\title{
Lactobacillus paracasei
}

National Cancer Institute

\section{Source}

National Cancer Institute. Lactobacillus paracasei. NCI Thesaurus. Code C123494.

A species of Gram-positive, rod shaped, facultatively heterofermentative bacteria in the phylum Firmicutes. This species is nonmotile and non spore forming. L. paracasei is a normal flora component of the the human oral cavity and gastrointestinal tract. It may be considered a subspecies of Lactobacillus casei. 